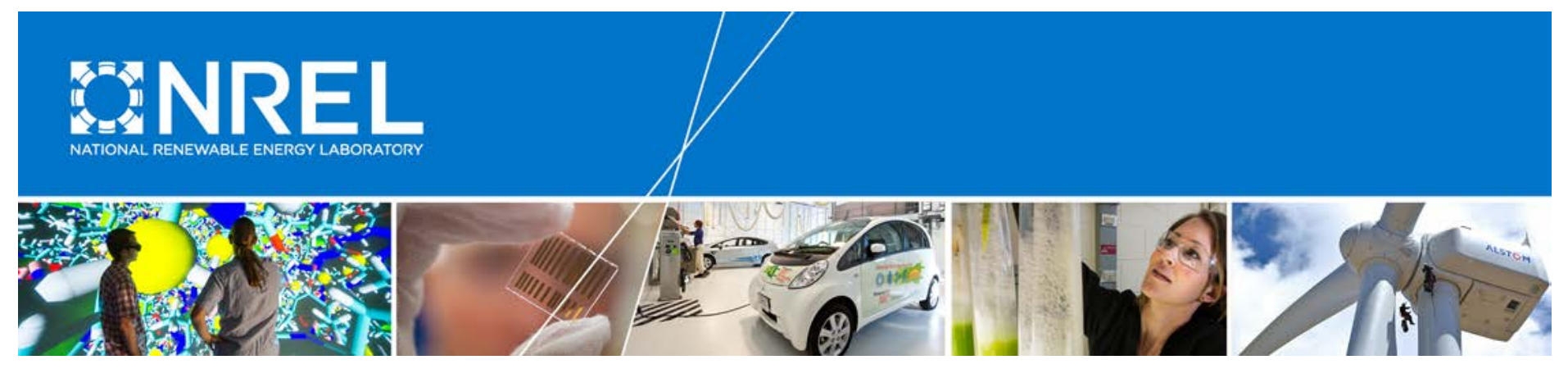

\title{
Stability Assessment of a System Comprising a Single Machine and Inverter with Scalable Ratings
}

Yashen Lin, Brian Johnson, and Vahan Gevorgian National Renewable Energy Laboratory

Victor Purba and Sairaj Dhople University of Minnesota

Presented at the IEEE North American Power Symposium September 17-19, 2017 Morgantown, West Virginia

NREL is a national laboratory of the U.S. Department of Energy Office of Energy Efficiency \& Renewable Energy Operated by the Alliance for Sustainable Energy, LLC

This report is available at no cost from the National Renewable Energy Laboratory (NREL) at www.nrel.gov/publications.

\section{Conference Paper}

NREL/CP-5D00-67974

August 2017 


\section{NOTICE}

This report was prepared as an account of work sponsored by an agency of the United States government. Neither the United States government nor any agency thereof, nor any of their employees, makes any warranty, express or implied, or assumes any legal liability or responsibility for the accuracy, completeness, or usefulness of any information, apparatus, product, or process disclosed, or represents that its use would not infringe privately owned rights. Reference herein to any specific commercial product, process, or service by trade name, trademark, manufacturer, or otherwise does not necessarily constitute or imply its endorsement, recommendation, or favoring by the United States government or any agency thereof. The views and opinions of authors expressed herein do not necessarily state or reflect those of the United States government or any agency thereof.

This report is available at no cost from the National Renewable Energy Laboratory (NREL) at www.nrel.gov/publications.

Available electronically at SciTech Connect http:/www.osti.gov/scitech

Available for a processing fee to U.S. Department of Energy and its contractors, in paper, from:

U.S. Department of Energy

Office of Scientific and Technical Information

P.O. Box 62

Oak Ridge, TN 37831-0062

OSTI http://www.osti.gov

Phone: 865.576.8401

Fax: 865.576.5728

Email: reports@osti.gov

Available for sale to the public, in paper, from:

U.S. Department of Commerce

National Technical Information Service

5301 Shawnee Road

Alexandria, VA 22312

NTIS http://www.ntis.gov

Phone: 800.553 .6847 or 703.605 .6000

Fax: 703.605.6900

Email: orders@ntis.gov 


\section{Stability Assessment of a System Comprising a Single Machine and Inverter with Scalable Ratings}

\author{
Yashen Lin, Brian Johnson, Vahan Gevorgian \\ National Renewable Energy Laboratory \\ Power Systems Engineering Center \\ Email: \{yashen.lin, brian.johnson, vahan.gevorgian\}@ nrel.gov
}

\author{
Victor Purba, Sairaj Dhople \\ Department of Electrical and Computer Engineering \\ University of Minnesota \\ Email: \{purba002, sdhople\}@umn.edu
}

\begin{abstract}
Synchronous machines have traditionally acted as the foundation of large-scale electrical infrastructures and their physical properties have formed the cornerstone of system operations. However, with the increased integration of distributed renewable resources and energy-storage technologies, there is a need to systematically acknowledge the dynamics of powerelectronics inverters-the primary energy-conversion interface in such systems-in all aspects of modeling, analysis, and control of the bulk power network. In this paper, we assess the properties of coupled machine-inverter systems by studying an elementary system comprised of a synchronous generator, three-phase inverter, and a load. The inverter model is formulated such that its power rating can be scaled continuously across power levels while preserving its closed-loop response. Accordingly, the properties of the machine-inverter system can be assessed for varying ratios of machine-to-inverter power ratings. After linearizing the model and assessing its eigenvalues, we show that system stability is highly dependent on the inverter current controller and machine exciter, thus uncovering a key concern with mixed machineinverter systems and motivating the need for next-generation grid-stabilizing inverter controls.
\end{abstract}

\section{INTRODUCTION}

Renewable portfolio standards, market trends, and increased consumer awareness have resulted in a pronounced increase in the integration of distributed renewable resources of energy in the power system. In contrast to conventional fossilfuel-driven generation which utilizes synchronous machines for energy conversion, renewable energy sources are fundamentally different in that they are typically coupled to the grid through power-electronics interfaces [1]. This trend broadly implies that next-generation power networks will be dominated by energy-conversion interfaces that offer limited-to-no mechanical inertia - a physical system property that currently underpins many operations and control tasks. Recognizing this generational change, in this paper, we propose a framework to characterize the small-signal stability of mixed machineinverter systems.

We investigate the small-signal stability of the system in Fig. 1 which consists of a synchronous machine and inverter serving a load. It should be noted that although practical systems contain large collections of interconnected machines grouped into balancing areas, we focus on this elemental system to glean insights that would otherwise be imperceptible were a more complex system investigated. With that being said, the main contribution of our work is that it addresses a critical challenge in modeling inverter-machine interactions: the large disparity in ratings between inverters and machines.

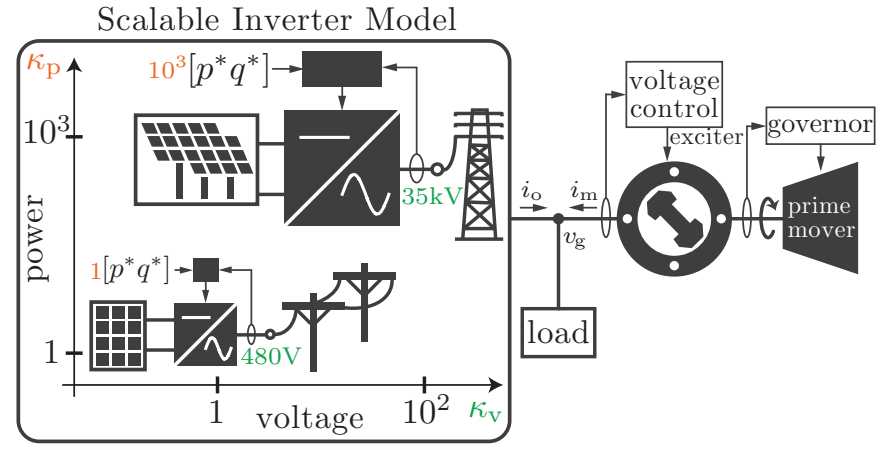

Fig. 1: Model of a single-machine single-inverter system, where the power and voltage ratings of the inverter are scalable. This model is an abstraction of low-inertia systems and is intended to assess possibly unforeseen dynamic interactions between machines and inverters as more inverters are installed in networks.

For instance, synchronous machines in a typical power plant are commonly rated for 100's of MVA while inverters are generally no larger than 100's of KVA. To address this challenge, we propose a dynamical scalable inverter model whose power and terminal-voltage ratings can be scaled to values representative in bulk power systems. We outline a procedure to do so that is grounded in systematically scaling model parameters, filter elements, and controller gains (with voltage- and power-scaling parameters $\kappa_{\mathrm{v}}$ and $\kappa_{\mathrm{p}}$ (see Fig. 1)). Increasing the values of these scaling parameters can capture one of two scenarios: i) connecting many small inverters in parallel to obtain a larger aggregate, or ii) designing an inverter with a higher power level from some known nominal specifications. The end result in either case is that we obtain an aggregated inverter model with power or voltage ratings that can be scaled to match (or even exceed) those of conventional synchronous machines. Tuning the values of these scaling parameters, we will study the combined machine-inverter dynamic model at varying levels of inverter penetration (i.e., varying levels inverter power ratings). To assess stability, we linearize the differential-algebraic-equation model and analyze the eigenvalues of the resulting linear model for scenarios such as: changing the mechanical inertia of the machine, disabling different control loops in the inverter, and changing controller gains. Note that we focus on prototypical inverter control strategies, which we call grid-following controls, to assess potential issues with business-as-usual approaches. Advanced inverter control strategies are beyond the scope of this paper. 
Prior work that relates to this effort includes classical results on the stability of interconnected synchronous generators in the bulk system [2]. Given that power electronics energyconversion interfaces have only recently been deployed in significant numbers and capacities approaching representative values in the bulk system, inverter dynamics have hitherto not been systematically acknowledged. There is a growing body of literature that has investigated the dynamics of (predominantly) inverter-based islanded microgrids [3]-[6]. With regard to the setting we study, i.e., grid-connected inverter systems, destabilizing interactions between inverters with conventional phaselocked-loops (PLL) have been recently uncovered and analyzed in [7]; small-signal stability of mixed inverter-machine microgrids as a function of inverter control gains has been studied in [8], and small-signal stability of a wind turbine-machine system has been investigated in [9]. While these works have characterized the dynamics of heterogeneous machine-inverter systems to some extent, the effect of inverter penetration level on stability has not been explicitly addressed. Finally, there are several large-scale system modeling studies that assess the impact of variable renewable generation on system dynamics [10]. However, inverters in such studies are typically modeled as real-power injections and the dynamical models that underly inverter operation are disregarded for analytical and computational convenience.

The remainder of this paper is organized as follows. The inverter and machine models are outlined in Section II, smallsignal stability analysis is performed in Section III, and concluding statements and directions for future work are outlined in Section IV.

\section{Single-MACHINE SCALABLE-INVERTER MOdEL}

In this section, we first describe the inverter and machine dynamical models in their local dq reference frames. Subsequently, we discuss the network model and how these three models are coupled.

\section{A. Machine Model}

We consider a prototypical steam-driven generator that consists of a synchronous machine, exciter, governor, and prime mover. Given that models for such systems are well established, we only provide a brief overview and refer interested readers to [2] for details. The model we consider consists of a frequency loop that captures the rotor dynamics and a voltage loop that encapsulates the electromagnetics. (See Fig. 2.) The frequency dynamics include those of the governor and turbine and they dictate the evolution of the generator rotor angle. The terminal voltage is governed by the voltage regulator and exciter circuitry. Without loss of generality, we assume the exciter is of type AC4A [2] which utilizes a first-order leadlag compensator. Stator- and damper-winding dynamics as well as power system stabilizer dynamics have been neglected in addition to nonlinearitites such as magnetic-saturation effects, voltage limiters, and frequency limiters.

The dynamics of the frequency- and voltage-control loops illustrated in Fig. 2 and discussed above can be succinctly captured in the following state-space model

$$
\dot{x}_{\mathrm{m}}=f_{\mathrm{m}}\left(x_{\mathrm{m}}, u_{\mathrm{m}}\right),
$$

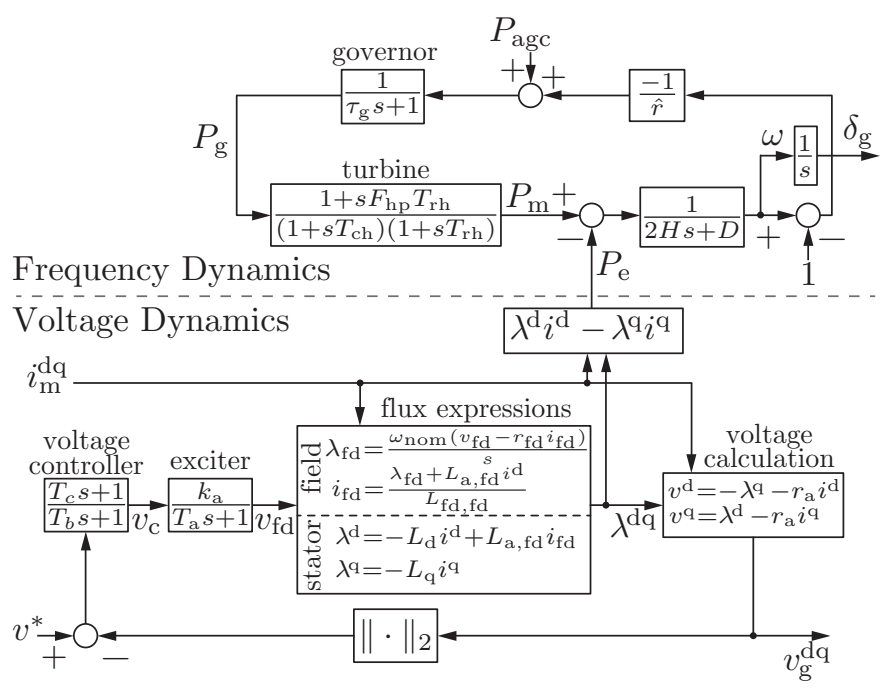

Fig. 2: Block diagram of a synchronous machine equipped with a prime mover, governor, and voltage excitation system.

with the following states and inputs:

$$
\begin{aligned}
& x_{\mathrm{m}}=\left[\delta_{\mathrm{g}}, \omega, P_{\mathrm{g}}, P_{\mathrm{gt}}, P_{\mathrm{m}}, v_{\mathrm{c}}, v_{\mathrm{fd}}, \lambda_{\mathrm{fd}}\right]^{\top}, \\
& u_{\mathrm{m}}=\left[P_{\mathrm{agc}}, v^{*}, i_{\mathrm{m}}^{\mathrm{dq}}\right]^{\top} .
\end{aligned}
$$

Above, $\delta_{\mathrm{g}}$ is the rotor angle (that establishes the dq reference frame), $\omega$ is the frequency, $P_{\mathrm{g}}$ is the governor output, $P_{\mathrm{gt}}$ is an internal steam turbine state, $P_{\mathrm{m}}$ is the mechanical power on the rotor, $v_{\mathrm{c}}$ is the voltage controller output, $v_{\mathrm{fd}}$ is the field voltage, and $\lambda_{\mathrm{fd}}$ is the field flux linkage. The inputs consist of the AGC reference signal, $P_{\text {agc }}$, the terminal voltage command $v^{*}$, and the machine terminal currents $i_{\mathrm{m}}^{\mathrm{dq}}=\left[i_{\mathrm{m}}^{\mathrm{d}}, i_{\mathrm{m}}^{\mathrm{q}}\right]^{\top}$.

Subsequently, we denote the machine power rating as $\bar{P}_{\mathrm{m}}$. Furthermore, all pertinent model parameters, inputs, and states are per-unitized using the machine power and voltage ratings as base values [2].

\section{B. Scalable Inverter Model}

The model adopted for the three-phase inverter is shown in Fig. 3. Physical components are the output $L C L$ filter and the three-leg voltage source inverter. Closed-loop controllers include a PLL, current controller, and power controller. The PLL synchronizes to the measured voltage $v_{\mathrm{o}}$, the power controller produces current references for the current controller, and the current controller yields the switch terminal voltage $v_{\mathrm{i}}$. From here forward, we utilize an averaged model where the PWM and switching dynamics are neglected and we assume that the voltage commands generated by the current controller appear at the switch terminals [11]. This control structure is well-established [4]; and building on it, we introduce the power and voltage scaling factors, denoted by $\kappa_{\mathrm{p}}$ and $\kappa_{\mathrm{v}}$, respectively, to yield a model that can accommodate tunable voltage and power ratings. Before delving into the details of the scaled inverter, we first briefly overview the dynamical model for the unscaled inverter.

The dynamics of the $L C L$ filter and control loops for an unscaled inverter (i.e., with the choice $\kappa_{\mathrm{v}}=\kappa_{\mathrm{p}}=1$ ) can be written compactly as:

$$
\dot{x}_{\mathrm{i}}=f_{\mathrm{i}}\left(x_{\mathrm{i}}, u_{\mathrm{i}}\right),
$$




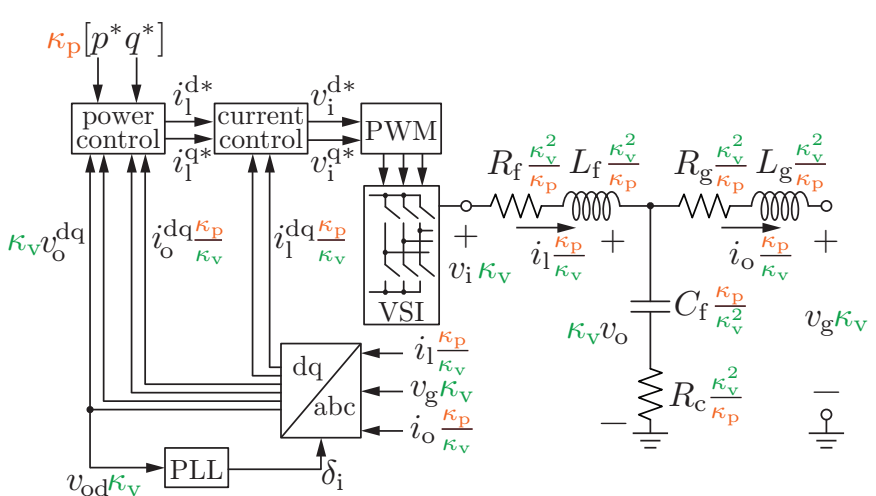

Fig. 3: Scalable three-phase inverter model with grid-following control. The power and voltage scaling factors are $\kappa_{\mathrm{p}}$ and $\kappa_{\mathrm{V}}$ respectively.

where the dynamical states and inputs are given by

$$
\begin{aligned}
& x_{\mathrm{i}}=\left[i_{1}^{\mathrm{dq}}, i_{\mathrm{o}}^{\mathrm{dq}}, v_{\mathrm{o}}^{\mathrm{dq}}, \gamma^{\mathrm{dq}}, p_{\mathrm{avg}}, q_{\mathrm{avg}}, \phi_{\mathrm{pq}}, v_{\mathrm{PLL}}, \phi_{\mathrm{PLL}}, \delta_{\mathrm{i}}\right]^{\top}, \\
& u_{i}=\left[p^{*}, q^{*}, v_{\mathrm{g}}^{\mathrm{dq}}\right]^{\top} .
\end{aligned}
$$

Above, $i_{1}^{\mathrm{dq}}=\left[i_{1}^{\mathrm{d}}, i_{1}^{\mathrm{q}}\right]^{\top}$ is the filter current, $i_{\mathrm{o}}^{\mathrm{dq}}=\left[i_{\mathrm{o}}^{\mathrm{d}}, i_{\mathrm{o}}^{\mathrm{q}}\right]^{\top}$ is the terminal current, $v_{\mathrm{o}}^{\mathrm{dq}}=\left[v_{\mathrm{o}}^{\mathrm{d}}, v_{\mathrm{O}}^{\mathrm{q}}\right]^{\top}$ is the filter voltage, $\gamma^{\mathrm{dq}}=\left[\gamma^{\mathrm{d}}, \gamma^{\mathrm{q}}\right]^{\top}$ captures the states for the current PI controller, $p_{\text {avg }}$ and $q_{\text {avg }}$ are the low-pass-filtered measurements of the inverter real and reactive power, $\phi_{\mathrm{pq}}=\left[\phi_{\mathrm{p}}, \phi_{\mathrm{q}}\right]^{\top}$ captures the states for the real and reactive power PI controllers, $v_{\mathrm{PLL}}$ and $\phi_{\mathrm{PLL}}$ are the filtered d-axis voltage measurement and the PI compensator state for the PLL, respectively, and $\delta_{\mathrm{i}}$ is the angle for the dq transformation. The input signals $p^{*}$ and $q^{*}$ are the unscaled real and reactive power set points, and $v_{\mathrm{g}}^{\mathrm{dq}}=\left[v_{\mathrm{g}}^{\mathrm{d}}, v_{\mathrm{g}}^{\mathrm{q}}\right]^{\top}$ is the unscaled grid voltage at the point of interconnection.

The dynamics in (4) are specified in dq coordinates with the PLL defining the inverter reference frame angle, $\delta_{\mathrm{i}}$. We provide a brief overview of the dynamics next. The PLL dynamics are given by

$$
\begin{aligned}
\dot{v}_{\mathrm{PLL}} & =\omega_{\mathrm{c}, \mathrm{PLL}}\left(v_{\mathrm{o}}^{\mathrm{d}}-v_{\mathrm{PLL}}\right), \\
\dot{\phi}_{\mathrm{PLL}} & =-v_{\mathrm{PLL}}, \\
\dot{\delta}_{\mathrm{i}} & =\omega_{\mathrm{nom}}-k_{\mathrm{PLL}}^{p} v_{\mathrm{PLL}}+k_{\mathrm{PLL}}^{i} \phi_{\mathrm{PLL}}:=\omega_{\mathrm{PLL}},
\end{aligned}
$$

where $\omega_{\mathrm{c}, \mathrm{PLL}}$ is the cutoff frequency of the filter for the $v_{\mathrm{o}}^{\mathrm{d}}$ measurement, $\omega_{\text {nom }}$ is the nominal ac system frequency, and the proportional-integral control gains are given by $k_{\mathrm{PLL}}^{p}$ and $k_{\mathrm{PLL}}^{i}$.

The real and reactive power delivered by the inverter are given by $p=3 / 2\left(v_{\mathrm{o}}^{\mathrm{d}} i_{\mathrm{o}}^{\mathrm{d}}+v_{\mathrm{o}}^{\mathrm{q}} i_{\mathrm{o}}^{\mathrm{q}}\right)$ and $q=3 / 2\left(v_{\mathrm{o}}^{\mathrm{q}} i_{\mathrm{o}}^{\mathrm{d}}-v_{\mathrm{o}}^{\mathrm{d}} i_{\mathrm{o}}^{\mathrm{q}}\right)$, respectively. The power controller dynamics are

$$
\dot{s}_{\text {avg }}=\omega_{\mathrm{c}}\left([p, q]^{\top}-s_{\text {avg }}\right), \dot{\phi}_{\mathrm{pq}}=\left[p^{*}, q^{*}\right]^{\top}-s_{\text {avg }},
$$

and the current commands that are derived from it are given by

$$
i_{\mathrm{l}}^{\mathrm{dq} *}=k_{\mathrm{PQ}}^{p} \dot{\phi}_{\mathrm{pq}}+k_{\mathrm{PQ}}^{i} \phi_{\mathrm{pq}},
$$

where $s_{\mathrm{avg}}=\left[p_{\mathrm{avg}}, q_{\mathrm{avg}}\right]^{\top}, i_{1}^{\mathrm{dq} *}=\left[i_{1}^{\mathrm{d} *}, i_{1}^{\mathrm{q} *}\right]^{\top}$, and the gains of the power controller are $k_{\mathrm{PQ}}^{p}$ and $k_{\mathrm{PQ}}^{i}$. Lastly, the current controller is specified by

$$
\dot{\gamma}^{\mathrm{dq}}=i_{1}^{\mathrm{dq} *}-i_{1}^{\mathrm{dq}},
$$

and its output which yields the switch terminal voltage is

$$
v_{\mathrm{i}}^{\mathrm{dq} *}=k_{\mathrm{i}}^{p} \dot{\gamma}^{\mathrm{dq}}+k_{\mathrm{i}}^{i} \gamma^{\mathrm{dq}}+\left[\begin{array}{cc}
0 & -1 \\
1 & 0
\end{array}\right] \omega_{\mathrm{PLL}} L_{\mathrm{f}} i_{\mathrm{l}}^{\mathrm{dq}},
$$

where $k_{\mathrm{i}}^{p}$ and $k_{\mathrm{i}}^{i}$ are the compensator gains and $L_{\mathrm{f}}$ is the inductance of the first branch of the $L C L$ filter (see Fig. 3). Finally, we assume that $v_{\mathrm{i}}^{\mathrm{dq}}=v_{\mathrm{i}}^{\mathrm{dq} *}$. The $L C L$ filter dynamics can be described using established methods [11] and further details of the controllers described above are in [4].

Next, we describe how the filter parameters and control gains of the unscaled inverter can be systematically tuned to obtain an inverter model for a desired terminal voltage and power level while preserving its closed-loop response (we clarify what this means shortly). Define the scaled power and voltage ratings $\bar{P}_{\mathrm{i}}:=\kappa_{\mathrm{p}} P_{\mathrm{i}}$ and $\bar{V}_{\mathrm{i}}:=\kappa_{\mathrm{v}} V_{\mathrm{i}}$, respectively, where $P_{\mathrm{i}}$ and $V_{\mathrm{i}}$ are the ratings for the original unscaled inverter. To obtain the scalable model with ratings $\bar{P}_{\mathrm{i}}$ and $\bar{V}_{\mathrm{i}}$, we modify the $L C L$ filter parameters and control gains as follows (also illustrated in Fig. 3):

- LCL filter: $\frac{\kappa_{\mathrm{v}}^{2}}{\kappa_{\mathrm{p}}} L_{\mathrm{f}}, \frac{\kappa_{\mathrm{v}}^{2}}{\kappa_{\mathrm{p}}} R_{\mathrm{f}}, \frac{\kappa_{\mathrm{v}}^{2}}{\kappa_{\mathrm{p}}} L_{\mathrm{o}}, \frac{\kappa_{\mathrm{v}}^{2}}{\kappa_{\mathrm{p}}} R_{\mathrm{O}}, \frac{\kappa_{\mathrm{p}}}{\kappa_{\mathrm{v}}^{2}} C, \frac{\kappa_{\mathrm{v}}^{2}}{\kappa_{\mathrm{p}}} R_{\mathrm{C}}$.

- Power controller: $k_{\mathrm{PQ}}^{p} / \kappa_{\mathrm{v}}, k_{\mathrm{PQ}}^{i} / \kappa_{\mathrm{v}}$.

- Current controller: $\frac{\kappa_{\mathrm{v}}^{2}}{\kappa_{\mathrm{p}}} k_{\mathrm{i}}^{p}, \frac{\kappa_{\mathrm{v}}^{2}}{\kappa_{\mathrm{p}}} k_{\mathrm{i}}^{i}$.

- $\quad$ PLL: $k_{\mathrm{PLL}}^{p} / \kappa_{\mathrm{v}}, k_{\mathrm{PLL}}^{i} / \kappa_{\mathrm{v}}$.

Let us denote the scaled inverter states as $x_{i}^{s}$, and suppose they are ordered the same way as in (5). With the $L C L$ filter elements and controller parameters scaled as discussed above, the dynamics of the scaled inverter can be described in the form

$$
\dot{x}_{\mathrm{i}}^{s}=f_{\mathrm{i}}^{s}\left(x_{\mathrm{i}}^{s}, u_{\mathrm{i}}^{s}\right),
$$

where the input $u_{\mathrm{i}}^{s}=\left[\kappa_{\mathrm{p}} p^{*}, \kappa_{\mathrm{p}} q^{*}, \kappa_{\mathrm{v}} v_{\mathrm{g}}^{\mathrm{dq}}\right]^{\top}$, and $f_{\mathrm{i}}^{s}(\cdot, \cdot)$ has the same structure as $f_{\mathrm{i}}(\cdot, \cdot)$. Algebraic manipulations of the inverter dynamical model described above reveal that

$$
x_{\mathrm{i}}^{s}(t)=\operatorname{diag}(\underline{\kappa}) x_{\mathrm{i}}(t), \forall t \geq t_{0},
$$

where $\underline{\kappa}:=\left[\frac{\kappa_{\mathrm{p}}}{\kappa_{\mathrm{v}}}, \frac{\kappa_{\mathrm{p}}}{\kappa_{\mathrm{v}}}, \kappa_{\mathrm{v}}, \frac{\kappa_{\mathrm{p}}}{\kappa_{\mathrm{v}}}, \kappa_{\mathrm{p}}, \kappa_{\mathrm{p}}, \kappa_{\mathrm{p}}, \kappa_{\mathrm{v}}, \kappa_{\mathrm{v}}, 1\right]^{\top}$ provided the initial conditions for the unscaled and scaled inverters at time $t_{0}$ are picked such that $x_{\mathrm{i}}^{s}\left(t_{0}\right)=\operatorname{diag}(\underline{\kappa}) x_{\mathrm{i}}\left(t_{0}\right)$. From the definition of the states in (5) and the property in (15), we see that in the scaled states $x_{\mathrm{i}}^{s}$, the power related states are scaled by the factor of $\kappa_{\mathrm{p}}$, the voltage related states are scaled by the factor of $\kappa_{\mathrm{v}}$, the current related states are scaled by the factor of $\kappa_{\mathrm{p}} / \kappa_{\mathrm{v}}$, and the angle $\delta_{\mathrm{i}}$ is not scaled. Lastly, all inverter model parameters, inputs, and states are per-unitized. The base values are the same as those used in the machine model.

\section{Network Model}

The inverter and machine models above are each represented in their respective coordinate frames. To collectively analyze these subsystems, we perform a coordinate transformation that places the machine terminal variables in the inverter reference frame as determined by the PLL angle, $\delta_{\mathrm{i}}$. Suppose quantities superscripted with $d, q$ are in the inverter $d q$ reference frame, and those superscripted by $\mathrm{D}, \mathrm{Q}$ are in 
TABLE I: Parameters of the machine model under consideration. All values are in per unit unless specified otherwise.

\begin{tabular}{c|c|c}
\hline$H=2.9 \mathrm{~s}$ & $D=1$ & $\hat{r}=0.05$ \\
\hline$\tau_{\mathrm{g}}=0.2 \mathrm{~s}$ & $F_{\mathrm{hp}}=0.3$ & $T_{\mathrm{rh}}=7 \mathrm{~s}$ \\
\hline$T_{\mathrm{ch}}=0.3 \mathrm{~s}$ & $k_{\mathrm{a}}=0.0745$ & $T_{\mathrm{a}}=0.04 \mathrm{~s}$ \\
\hline$T_{\mathrm{b}}=12 \mathrm{~s}$ & $T_{\mathrm{c}}=1 \mathrm{~s}$ & $R_{\mathrm{fd}}=0.0006$ \\
\hline$R_{\mathrm{a}}=0.003$ & $L_{\mathrm{a}, \mathrm{fd}}=1.66$ & $L_{\mathrm{fd}, \mathrm{fd}}=1.825$ \\
\hline$L_{\mathrm{d}}=1.81$ & $L_{\mathrm{q}}=1.76$ & $P_{\mathrm{agc}}=0.9 \mathrm{pu}$ \\
\hline
\end{tabular}

the machine $d q$ reference frame. Accordingly, the following transformation

$\left[\begin{array}{l}x_{\mathrm{m}}^{\mathrm{d}} \\ x_{\mathrm{m}}^{\mathrm{q}}\end{array}\right]=\left[\begin{array}{cc}\cos \left(\omega_{\mathrm{nom}}\left(\delta_{\mathrm{g}}-\delta_{\mathrm{i}}\right)\right) & -\sin \left(\omega_{\text {nom }}\left(\delta_{\mathrm{g}}-\delta_{\mathrm{i}}\right)\right) \\ \sin \left(\omega_{\mathrm{nom}}\left(\delta_{\mathrm{g}}-\delta_{\mathrm{i}}\right)\right) & \cos \left(\omega_{\mathrm{nom}}\left(\delta_{\mathrm{g}}-\delta_{\mathrm{i}}\right)\right)\end{array}\right]\left[\begin{array}{c}x_{\mathrm{m}}^{\mathrm{D}} \\ x_{\mathrm{m}}^{\mathrm{Q}}\end{array}\right]$,

converts variables from the machine to inverter frame, where $\delta_{\mathrm{g}}-\delta_{\mathrm{i}}$ is the angle difference between the machine rotor and inverter PLL.

We suppose that the network variables are described as phasor quantities in the inverter frame. From Kirchhoff's laws, we have:

$$
V_{\mathrm{g}}=z\left(I_{\mathrm{m}}+I_{\mathrm{i}}\right)
$$

where $z$ is the complex load impedance, $I_{\mathrm{m}}=i_{\mathrm{m}}^{\mathrm{d}}+j i_{\mathrm{m}}^{\mathrm{q}}$ and $I_{\mathrm{i}}=i_{\mathrm{o}}^{\mathrm{d}}+j i_{\mathrm{o}}^{\mathrm{q}}$ are the terminal machine and inverter currents, respectively, and $V_{\mathrm{g}}=v_{\mathrm{g}}^{\mathrm{d}}+j v_{\mathrm{g}}^{\mathrm{q}}$ is the machine terminal voltage. Combining (16) and (17) the network equations can be captured by the general algebraic model:

$$
0=g\left(i_{\mathrm{m}}^{\mathrm{dq}}, i_{\mathrm{o}}^{\mathrm{dq}}, v_{\mathrm{g}}^{\mathrm{dq}}, \delta_{\mathrm{g}}-\delta_{\mathrm{i}}\right) .
$$

Having established the coupled inverter-machine dynamical model, we assess its small-signal stability as we vary the inverter penetration level, which we define as the ratio

$$
\frac{\bar{P}_{\mathrm{i}}}{\bar{P}_{\mathrm{i}}+\bar{P}_{\mathrm{m}}},
$$

where $\bar{P}_{\mathrm{i}}=\kappa_{\mathrm{p}} P_{\mathrm{i}}$, with $P_{\mathrm{i}}$ being the power rating of the unscaled inverter model, and $\kappa_{\mathrm{p}}$ the power-scaling parameter.

\section{SMALL-SIGNAL STABILITy ANALYSIS}

In this section, we examine a linearized version of the coupled machine-inverter system described by the models in Section II. After investigating instabilities in the full-order system model, we uncover the root cause of the observed instabilities. Towards that end, we bypass and/or modify various subsystems within the inverter and machine models to assess the impact of each. Ultimately, we will show that for the particular models under consideration, interactions between the machine voltage exciter and inverter current controller lead to small-signal instability at high levels of inverter penetration.

\section{A. Linearization}

The dynamical models (1), (14), and the algebraic constraint given by (18) collectively yield a differential algebraic equation network model. Utilizing the coupling among $i_{\mathrm{m}}^{\mathrm{dq}}$, and $\lambda_{\mathrm{fd}}$ (see Fig. 2), we solve for $v_{\mathrm{g}}^{\mathrm{dq}}$ and $i_{\mathrm{m}}^{\mathrm{dq}}$ from (18) in
TABLE II: Parameters of the inverter model utilized in the case-study.

\begin{tabular}{c|c|c}
\hline$L_{\mathrm{f}}=1 \mathrm{mH}$ & $R_{\mathrm{f}}=0.7 \Omega$ & $L_{\mathrm{o}}=0.2 \mathrm{mH}$ \\
\hline$R_{\mathrm{O}}=0.12 \Omega$ & $C=24 \mu \mathrm{F}$ & $R_{\mathrm{c}}=0.02 \Omega$ \\
\hline$k_{\mathrm{PQ}}^{p}=0.01(\mathrm{~V})^{-1}$ & $k_{\mathrm{PQ}}^{\imath}=0.1(\mathrm{~V} \cdot \mathrm{s})^{-1}$ & $k_{\mathrm{i}}^{p}=16.4 \mathrm{~V} / \mathrm{A}$ \\
\hline$k_{\mathrm{i}}^{\imath}=30.4 \mathrm{~V} /(\mathrm{A} \cdot \mathrm{s})$ & $k_{\mathrm{PLL}}^{p}=0.25 \mathrm{rad} / \mathrm{V}$ & $k_{\mathrm{PLL}}^{2}=2 \mathrm{rad} /(\mathrm{V} \cdot \mathrm{s})$ \\
\hline
\end{tabular}

terms of the states $\lambda_{\mathrm{fd}}, i_{\mathrm{o}}^{\mathrm{dq}}, \delta_{\mathrm{g}}-\delta_{\mathrm{i}}$. Then we can we obtain an augmented dynamical system from (1) and (14):

$$
\dot{x}=f(x, u),
$$

where $x$ collects all states in $x_{\mathrm{i}}^{s}$ and $x_{\mathrm{m}}$, except replacing $\delta_{\mathrm{g}}$ and $\delta_{\mathrm{i}}$ with their difference $\delta_{\mathrm{g}}-\delta_{\mathrm{i}}$, and $u=$ $\left[P_{\text {agc }}, v^{*}, \kappa_{\mathrm{p}} p^{*}, \kappa_{\mathrm{p}} q^{*}\right]^{\top}$ collects all the exogenous inputs to the system. Linearizing around the equilibrium point $\left(x^{*}, u^{*}\right)$, we obtain

$$
\Delta \dot{x}=A \Delta x+B \Delta u,
$$

where $A=\left.\frac{\partial f}{\partial x}\right|_{\left(x^{*}, u^{*}\right)}$ and $B=\left.\frac{\partial f}{\partial u}\right|_{\left(x^{*}, u^{*}\right)}$.

\section{B. Case Study Description}

The machine under consideration has a power rating of $\bar{P}_{\mathrm{m}}=555 \mathrm{MVA}$, line-to-line voltage rating of $24 \mathrm{kV}$, and a nominal frequency of $\omega_{\text {nom }}=2 \pi \times 60 \mathrm{rad} / \mathrm{s}$. (These serve as the base values.) Some relevant machine parameter values and inputs are listed in Table I (these are adopted from [2]). The load impedance is calculated such that it consumes $500 \mathrm{MW}$ and $50 \mathrm{MVAR}$ at the nominal voltage. The parameters for the unscaled inverter are given in Table II and correspond to a custom-built hardware prototype at the National Renewable Energy Laboratory with power and lineto-neutral voltage ratings of $P_{\mathrm{i}}=1 \mathrm{~kW}$ and $V_{\mathrm{i}}=120 \mathrm{~V}_{\mathrm{LN}}$, respectively. The inverter voltage scaling factor is selected as $\kappa_{\mathrm{v}}=24 \times 10^{3} /(\sqrt{3} 120)$ so that the inverter terminal voltage is compatible with the rated machine voltage. For the ensuing analysis, we vary the inverter real power reference, $\kappa_{\mathrm{p}} p^{*}$, via $\kappa_{\mathrm{p}}$ such that it is equal to the inverter power rating, $P_{\mathrm{i}}$, when the rating is less $250 \mathrm{MW}$ (half of the load) and $\kappa_{\mathrm{p}} p^{*}$ remains fixed at $250 \mathrm{MW}$ when $\bar{P}_{\mathrm{i}}>250 \mathrm{MW}$.

\section{Subsystem and Root-cause Analysis}

In this section, we will either modify or bypass each pertinent subsystem in the machine-inverter system and study the effect on small-signal system stability (by computing the eigenvalues of the matrix $A(21)$ ). We begin with a nominal base case.

1) Nominal Case: For the nominal parameters given above, we increase the inverter power scaling factor, $\kappa_{\mathrm{p}}$, in small increments, and compute the equilibrium point from (20) and eigenvalues of the system matrix $A$ in (21). In Fig. 4, we plot the real part of the eigenvalue with the largest real part, denoted as $\max (\operatorname{Re}(\lambda))$, as a function of the inverter penetration level (19), where $\lambda$ is the vector of complex eigenvalues of the matrix $A$. If $\max (\operatorname{Re}(\lambda))>0$, the smallsignal model is unstable. As shown in Fig. 4, small-signal stability is maintained for inverter penetration levels below approximately $50 \%$. 


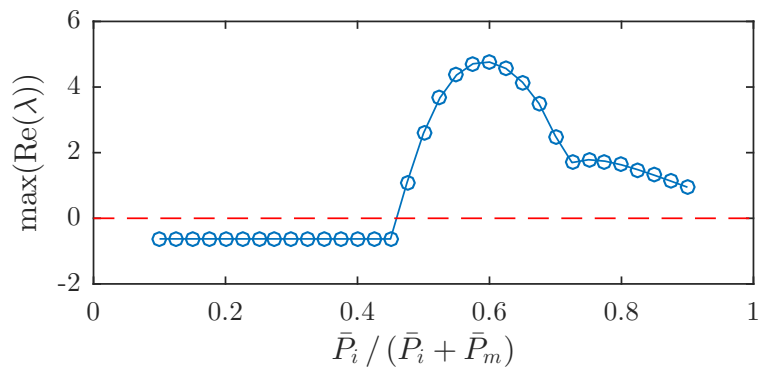

Fig. 4: Nominal case: Small-signal stability is ensured for penetration levels approaching $50 \%$.

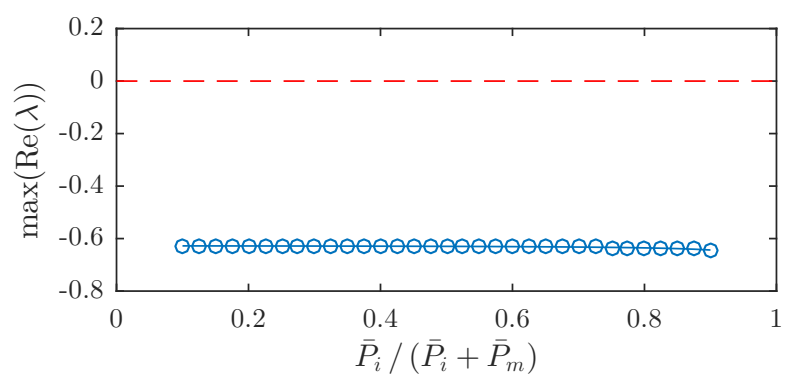

Fig. 5: Bypassing the machine AVR and exciter circuit significantly improves stability margins.

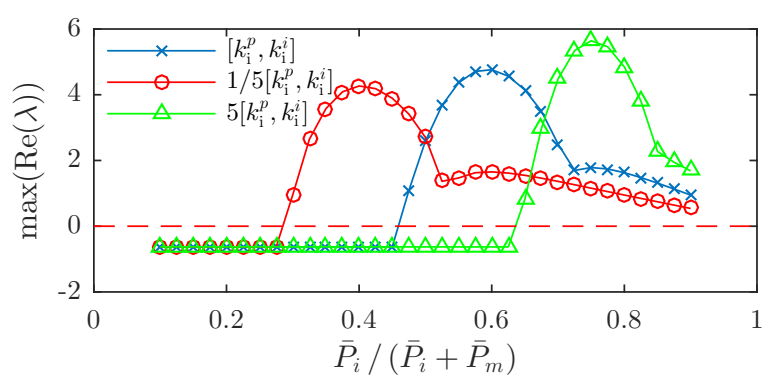

Fig. 6: Reduced current-controller gains adversely impact small-signal stability.

2) Machine Exciter: The machine terminal voltage is the signal that the inverter synchronizes to as it injects a controlled current. Accordingly, interactions between automatic voltage regulator (AVR) and associated exciter circuitry within the machine and the inverter controllers may be critical for system stability. To determine whether this is the case, we bypass the AVR and exciter circuit dynamics in the machine model and fix the machine voltage amplitude to its the nominal value. Figure 5 plots $\max (\operatorname{Re}(\lambda))$ as a function of the inverter penetration level (19) for this setting, and we see that smallsignal stability of the machine-inverter system is preserved across the full range of inverter penetration levels considered. Remarkably, stability is retained even with an inverter rating 10 times larger than the machine rating. This finding indicates that the presence of the AC4A-type AVR considered here has a major impact on the stability of machine-inverter systems and to the authors' knowledge is not reported in prior literature.

3) Current Controller: Next, we investigate impacts of the inverter current control gains on system stability. Here, the fullorder model is considered and we modify the current control

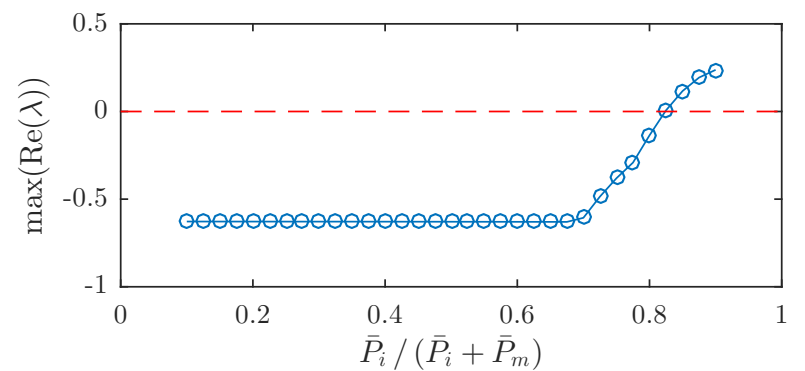

Fig. 7: Bypassing the PLL (assuming $\delta_{\mathrm{i}}=\delta_{\mathrm{m}}$ ) guarantees stability for penetration levels greater than $80 \%$.

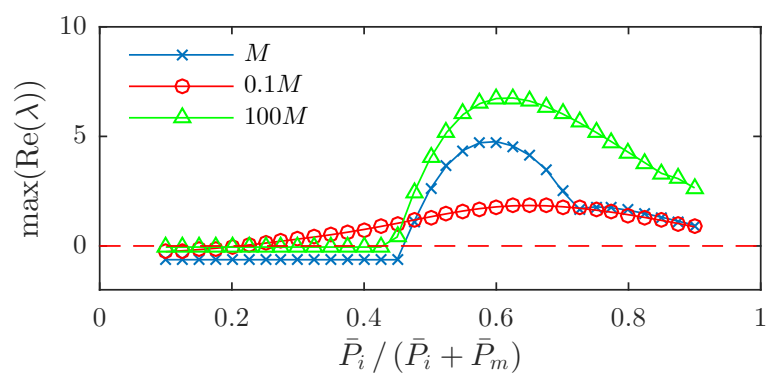

Fig. 8: System eigenvalues after modifying the machine rotor inertia.

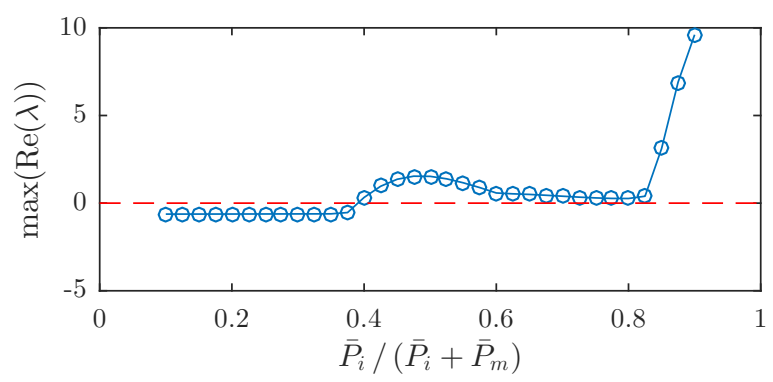

Fig. 9: Eigenvalues with the inverter power controller bypassed.

gains, $k_{\mathrm{i}}^{p}$ and $k_{\mathrm{i}}^{i}$, from the baseline values in Table II. In particular, Fig. 6 illustrates results for the case where $k_{\mathrm{i}}^{p}$ and $k_{\mathrm{i}}^{i}$ are scaled by a factor of 5 and 1/5 from the nominal value. As shown, small-signal instability is encountered at a smaller penetration level of approximately $30 \%$ when the gains are decreased by a factor of $1 / 5$, and an increased penetration level of $65 \%$ when the gains are increased by a factor of 5 . Hence, more aggressive current control gains increase the range of stable penetration levels.

4) PLL: The PLL is responsible for synchronizing the inverter with the terminal ac voltage and its performance impacts downstream inverter controllers. Therefore, it is plausible that it would play a major role in system stability. To explore this, we bypass the PLL by assuming that the inverter has perfect knowledge of the rotor angle (i.e., $\delta_{\mathrm{i}}=\delta_{\mathrm{g}}$ ). Although this operating condition is not physically possible, this assumption is a simple way to investigate the impact of the PLL on small-signal stability. As shown in Fig. 7, the system with no PLL and ideal angle-tracking at the inverter has a significantly enlarged region of stability. 


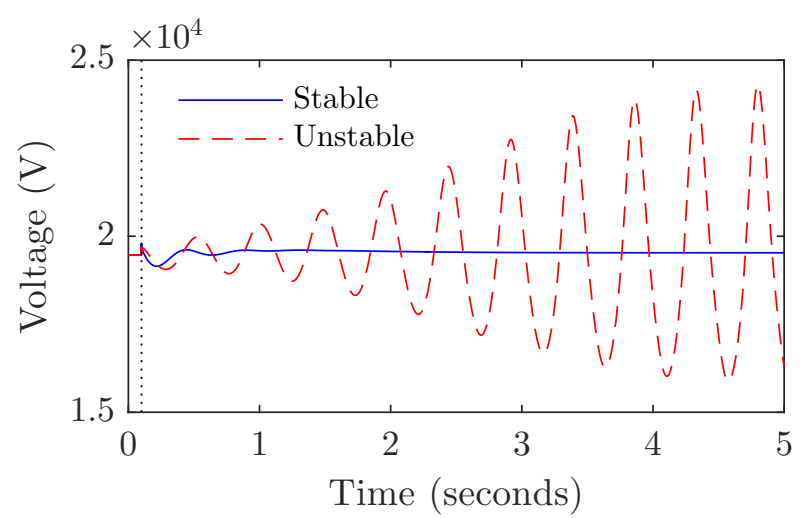

Fig. 10: Time domain response of the original nonlinear model. The blue solid line represents a stable case where inverter penetration level of $44 \%$; the red dashed line represents an unstable case where inverter penetration level of $55 \%$.

5) Machine frequency loop: Given that the inertia of the rotor system has a direct impact on system frequency dynamics, the impact of varying levels of system inertia on powersystem dynamic performance has recently attracted significant attention [12]. With this in mind, in Fig. 8 we plot $\max (\operatorname{Re}(\lambda))$ as a function of the inverter penetration level (19) when the inertia of the machine is scaled by factors of $1 / 10$ and 100 . While the stability margins are improved at higher inertia levels, surprisingly, these large variations in rotor inertia do not yield correspondingly large impacts on small-signal system stability.

6) Power controller: Lastly, we disable the inverter power controller by fixing the current reference values, $i_{1}^{\mathrm{dq} *}$, that are provided to the inverter inner current controller. As demonstrated in Fig. 9, we observe that the eigenvalues are similar to the base case and, accordingly, we conclude that the power controller has minimal impact on system stability.

\section{Validation of Small-signal Model}

In an attempt to validate the small-signal model, we consider two time-domain models where in each case $\max (\operatorname{Re}(\lambda))$ is either positive or negative, and in both cases the system is given a small perturbation in the form of a 5\% load step at $t=0.1 \mathrm{~s}$. Given that we are particularly interested in validating penetration levels near the instability boundary, we focus our time-domain models at operating conditions near this transition point. Starting at an equilibrium point and following the aforementioned load step, the responses in Fig. 10 illustrate the machine terminal voltage response at stable and unstable penetration levels of $44 \%$ and 55\%, respectively, from Fig. 4. As confirmed in the time-domain plots, the nonlinear dynamical model is indeed stable and unstable when $\max (\operatorname{Re}(\lambda))$ is negative and positive, respectively, thus validating the smallsignal model.

\section{CONCLUSION AND FUTURE WORK}

In this paper, we evaluated the stability of a coupled machine-inverter system as a function of the ratio of inverterto-machine power ratings. To enable this, we obtained a scalable inverter model where the control gains and physical parameters of a baseline inverter model are scaled as a function of voltage and power ratings. The scalable inverter model allowed us to formulate a system model with a tunable inverter rating level. After linearizing and analyzing the eigenvalues of the system, it was found that the AVR and excitation system of the machine exhibits a destabilizing interaction with the inverter current controllers. Furthermore, the gains of the inverter current controllers have a large impact on the stability of the machine-inverter system. We believe the analysis reported in this paper serves as a first step to charactering the stability of a power system with significant inverter-based generation. Future work includes extending this analytical framework to investigate multi-inverter multi-machine systems.

\section{ACKNOWLEDGMENT}

All authors were supported by the U.S. Department of Energy (DOE) Solar Energy Technologies Office under Contract No. DE-EE0000-1583. Y. Lin, B. Johnson, and V. Gevorgian were also supported by the DOE under Contract No. DEAC36-08-GO28308 with NREL; and S. Dhople was also supported by the National Science Foundation under the CAREER award, ECCS-1453921, and grant ECCS-1509277.

\section{REFERENCES}

[1] J. A. Taylor, S. V. Dhople, and D. S. Callaway, "Power systems without fuel," Renewable and Sustainable Energy Reviews, vol. 57, pp. 13221336, 2016.

[2] P. Kundur, N. J. Balu, and M. G. Lauby, Power system stability and control. McGraw-hill New York, 1994, vol. 7.

[3] N. Pogaku, M. Prodanovic, and T. C. Green, "Modeling, analysis and testing of autonomous operation of an inverter-based microgrid," IEEE Transactions on Power Electronics, vol. 22, no. 2, pp. 613-625, March 2007.

[4] M. Rasheduzzaman, J. A. Mueller, and J. W. Kimball, "Reducedorder small-signal model of microgrid systems," IEEE Transactions on Sustainable Energy, vol. 6, no. 4, pp. 1292-1305, 2015.

[5] J. Guerrero, L. de Vicuna, J. Matas, M. Castilla, and J. Miret, "A wireless controller to enhance dynamic performance of parallel inverters in distributed generation systems," IEEE Transactions on Power Electronics, vol. 19, no. 5, pp. 1205-1213, Sept. 2004.

[6] J. W. Simpson-Porco, F. Dörfler, and F. Bullo, "Synchronization and power sharing for droop-controlled inverters in islanded microgrids," Automatica, vol. 49, no. 9, pp. 2603-2611, 2013.

[7] D. Dong, B. Wen, D. Boroyevich, P. Mattavelli, and Y. Xue, "Analysis of phase-locked loop low-frequency stability in three-phase gridconnected power converters considering impedance interactions," IEEE Transactions on Industrial Electronics, vol. 62, no. 1, pp. 310-321, 2015.

[8] F. Katiraei, M. Iravani, and P. Lehn, "Small-signal dynamic model of a micro-grid including conventional and electronically interfaced distributed resources," IET Generation, Transmission \& Distribution, vol. 1, no. 3, pp. 369-378, 2007.

[9] J. Tan, X. Wang, Z. Chen, and M. Li, "Impact of a direct-drive permanent magnet generator (DDPMG) wind turbine system on power system oscillations," in Power and Energy Society General Meeting, 2012 IEEE. IEEE, 2012, pp. 1-8.

[10] N. W. Miller, B. Leonardi, R. DAquila, and K. Clark, "Western wind and solar integration study phase 3A: low levels of synchronous generation," NREL/TP-5D00-64822. Golden, Colorado: National Renewable Energy Laboratory, Tech. Rep., 2015.

[11] A. Yazdani and R. Iravani, Voltage-sourced converters in power systems. modeling, control, and applications. John Wiley \& Sons, 2010.

[12] A. Ulbig, T. S. Borsche, and G. Andersson, "Impact of low rotational inertia on power system stability and operation," IFAC Proceedings Volumes, vol. 47, no. 3, pp. 7290-7297, 2014. 